\title{
The Money Games Physicists Play
}

An article by econophysicist Yi-Cheng Zhang on page 51 describes some of his methods of modelling financial business. Below, Marcel Ausloos, a rival model maker, has his own views on what econophysicists should be doing

First, let us point out that this comment should not be considered as a criticism on or reply to that remarkable piece of writing by Zhang on page $51^{1}$. Rather, it is a set of random thoughts in view of the coherent set of works and findings in which our research group here at Liège in Belgium has recently been involved.

Since we are talking about money, it is necessary to recall that there are always two sides to a coin. Another point of view than the one offered by Zhang might be presented, and a more optimistic though realistic one. There is no perfect view though, and if we tossed a coin 100 hundred times most likely there would be approximately 50 Zhang faces to be observed.

There is so much data available on financing, banking, markets, stocks and currency exchange rates, discount and interest rates; and there are so many levels of observation: individual income(s), individual expenses, checking accounts and savings, number of public or private accounts, volumes going in and out and remaining still, debts and credits, tellers, dealers, bank outlets, businesses, governments; so many, that one is immediately tempted to play statistics, hopefully expecting some understanding of financial matters. (And who does not want to be rich?) There is so much data at so many different levels that any statistical physicist realizes that fractals ${ }^{2}$ play a role.

Fractals are unusual mathematical objects which have no scale. Though sometimes these objects are beautiful, too, ${ }^{3}$ maybe because they have no scale, maybe because they have some intrinsic symmetry, maybe because they have some basic symmetry, maybe because they are how nature works, ${ }^{, 4}$ or maybe because they might reveal what is unknown to us physicists and philosophers today. They bring new insights, new ways of approaching the world, the universe, and raise splendid new questions $s^{5}$. Sometimes one feels that rather than being limited to 'how' one is reaching the border of 'why?' - Why are we at the border of chaos and order? Maybe through fractals it will be possible to reach (literally) other universes than the one we have at hand today.

Fractals give both qualitative and quantitative answers ${ }^{6}$. This is so because one has much available data, because one can ask simple questions and draw simple (log-log) plots, because the functions one is dealing with are 'homogeneous' and because 'things' are 'self-similar'. That is universality. That is philosophy and physics merging together.

This is, therefore, the origin of the great

\section{L'argent ne fait pas le bonheur}

\section{Money doesn't bring you happiness Well known proverb}

desire and enthusiasm of physicists to compete with mathematicians who are involved with economists and economics. Physicists who know the realm of their models and the limits of their understanding, being honestly questioning at all levels their findings, have the possibility of bringing to economics much of their knowledge and the full paraphernalia of their tricks, theoretical or experimental.

Such physicists, born in thermodynamics, irreversibly growing up in the statistical physics community, have used from the very beginning the famous law of the least effort?: the minimum of free energy, the least entropic growth rate. They invent the minimal models, the less complicated ones. (Are these physicists lazy? Or rather, are they efficient?)

I do not believe that we will immediately supersede mathematicians in the Economics Faculties of the MBA Schools. We are physicists and too inclined to find new models (too quickly sometimes). But surely we are right to do so, to want to go beyond the statistics and ask 'how' by building a model. We knew coherence effects, correlation lengths, relaxation times, many body interactions, grand canonical ensembles, spins, phase transitions, critical exponents, mean field approximations and the renormalization group, cellular automata, computer games, and so on.

We were intrigued by "our human evolution"; we discovered self-organized criticality, ${ }^{9}$ earthquakes, fractures, biological and ecological distributions of populations, crystal growth, polymers and gels, dendrites, traffic jams, time and space dependent signals for soil or cloud or heart or brain or whatever. We could manipulate our computers to make virtual experiments. We played with mathematical techniques, we observed scaling and log periodic oscillations, ${ }^{10}$ we guessed their origin. ${ }^{11}$ We could find something in noise, in chaos there was regularity.12,13 We found that economic crashes could be predicted, ${ }^{14}$ as long as we had enough data. We observed the similarity with respect to a phase transition, ${ }^{15}$ and could even present the basic models, just as in ordinary statistical physics, because there are known critical exponents.

'Cooperative effects' was, for us, the name of the recent financial bubbles. Spin orientation, nearest neighbour interactions, and vortices ${ }^{16}$ were the signature of findings to which one could literally connect the real world. Money. Money. Money ${ }^{17}$.

Such cooperative effects are similar to those studied and implemented in evolution and ecology studies. Let us briefly recall two basic lines of thought in evolution: Darwin versus Lamarck ${ }^{18}$. For Darwin, "natural selection means the survival of the fittest, the preservation of variations as they arise and are beneficial to the being under its conditions of life". For Lamarck, the fittest is one day superseded by those who wish to survive and adapt. There is a sort of continuous cycling at the top with long or short time spans of stability. For Darwin, growth is almost intrinsic to evolution. However, infinite growth is not, of course, possible because there will be necessarily some levelling off because of the finite limit of resources, or even some drastic and rapid collapse. The same can be true and is known in economic life for small or large companies or economic (political) systems.

The evolution of humanity is of fundamental relevance. It has ecological conditions, but which are closely related to economic conditions. From an overall point of view, there is no need to quote all the per- 
tinent papers at this time, the field is blooming, there are already review papers, books ${ }^{19}$ and journals ${ }^{20}$ devoted to 'econophysics'.

Zhang claims that there are too many directions for investigation which will need research. On the contrary, Stauffer ${ }^{19}$ thinks that some self-organized [sic] framework will emerge; I believe so as well. I am, on this point, rather optimistic.

In our group at Liège we have touched on several points, covering by intellectual default and by scientific necessity several subjects. They may be considered points of interest as follows. As a premise let us point out that there are roughly two types of themes in econophysics at this time: stock markets and foreign currency exchange rates; these are based on different goals and strategies for 'players' (in other words 'traders'). With Vandewalle, we worked on both subjects.

Many other issues can be addressed:

- the creation of models based on financial insights and mathematical principles

- the calibration of models based on market information

- the simulation of models using specific algorithms

- model updating in line with market developments, and

- implementation of models in the form of 'black boxes' by practitioners

After pioneering work by well-known physicists in the realm of economics ${ }^{21}$, we got in touch with several banks and brokers - without much success except for getting our hands on much data. So, we turned to the scientific literature, and we also submitted to Europhys. Lett. a paper on the evolution of 23 foreign exchange currency rates (with respect to the DEM and BEF, for both emerging markets and well controlled ones). ${ }^{22}$

It was possible to show that the behaviour looks like turbulence, a fluid with vortex states; this was done through a fractallike analysis. Moreover, exchange rates seem to belong to different 'categories' depending on the money involved: strictly regulated European, hard dollar zone, emerging market.

One very relevant item, which greatly pleases fractalists and statistical physicists, is the discovery (after several attempts) that the markets are not really efficient (in the definition used by economists: whether models work). In almost all economics classrooms, teachers (if they don't rely on Peters's book ${ }^{23}$ ) assume a Gaussian - they seem to forget that the Bachelier analysis and the Gaussian hypothesis are not true. Mantegna et $\mathrm{al}^{24}$ have showed that the Levy

distribution is the true one, not the Gaussian one, for describing fluctuation correlations on the Standard \& Poor 500 (a New York Stock Exchange index). In fact, using a 'detrended fluctuation analysis method', as used by Stanley's group ${ }^{25}$ for sorting out DNA coding and noncoding regions, we showed in Physica $A^{26}$ that the time dependent signal for the foreign exchange currency rates does not obey the Brownian motion rule (coin tossing), but is rather a fractional Brownian motion (invented by Mandelbrot ${ }^{2}$ ); the 'diffusion' does not take place as the square root of time, but has an exponent quite different from 0.5 .

The most important point follows from this: predictability is possible. We will submit for publication a paper ${ }^{27}$ on this. Banks (and we spent a long time trying to persuade one Belgian bank) have not found our methods predictive enough. But we are sure that the physics community will better appreciate the fact that correlations are useful ways of obtaining positive returns

\section{"L'amour de la science et l'amour de l'argent se rencontrent rarement."}

\section{"The love of science and the love of money rarely come together." G. Herbert, 1651}

through strict data information feedback. It should be pointed out that the theory works this time as long as there is no external field - however, it can be shown that the external field of physicists in random walk or phase transition studies is very similar to the external field of economics ( $i e$ "Mr.

Greenspan comments on changing the interest rates"). It leads to a modification of the entropy dimension of the signal. ${ }^{26}$

It has been shown that a financial crisis occurs in currency exchange rate markets when the system loses its self-control (due to a complicated hierarchical structure ranging from big brokers to small businesses, through politicians, public and private actors). That can be useful in economic policy planning, certainly nowadays with deregulation, and globalization concepts. This raises new problems and has spurred on competition between banks and financial institutions. The development of very complex financial instruments and financial infrastructures has led to new challenges for many regulatory bodies.

What is interesting is that one can develop a winning strategy in a predictable situation. By actually betting on our principles, we virtually increased our chosen input capital by a factor of 40 during a 16 year period playing on the USD/DEM daily closing rate, and achieved less on the price of gold. ${ }^{27}$ However, this profit is only possible when there is no tax and no broker fee involved. However, this should suggest to economists a new approach in dealing with customers, like a bonus-malus working situation, or a flat fee, or an insurance rate like compromise and/or deal. These suggest the search for advanced valuation and risk assessment models and systems which quantify the returns and risks for financial institutions and investors. Physicists can certainly invent models and make proposals on that and the latest report by Bouchaud ${ }^{28}$ is the best proof that this approach will have a great future.

Fundamental questions remain unanswered: how do we best pace financial market development, or choose the optimal structure of the financial sector (banking versus securities etc) for our model? Studies from microstructure to macro policy responses will be useful here.

At this time, we do not have to make a living in the field; it is understandable that we can only play with pocket money, since most physicists have a wife, children, and pets, who should be considered first. After that, we can start playing probabilities and win and lose with some regularity.

Coming back to real physics, one should recall the historical idea of the sand pile model (the paradigm of self-organized criticality). In that spirit, we studied the $80-87$ and 90-97 stock market index, including the Dow Jones Industrial Average and the S\&P-500. It was possible to show that the signal follows a simple law, ${ }^{15}$ ie a logarithmic divergence on which a set of oscillations is superimposed due to finite scaling effects (it is equivalent in phase transitions to a complex critical exponent for which the real part $=0$ ).

This helped with predicting last year's market turmoil. We could predict the date and the amplitude of the crash. We predicted an upper boundary as early as August, and the October 27 date just the week before. ${ }^{29,30}$ As an 'award' for our achievements we appeared on TV in Belgium. It was not much, but it was recognition for physics in the world of the media.

With such a study we can show that the Asian markets behave differently from the 
European and American markets. This is obvious to economists. But consider this: when we discussed biological evolution with biologists they knew that Darwin was right. Nevertheless, Darwin did not have fractals to hand. Was it necessary or not to formulate and to model in abstract terms the Darwinian evolution? It's an interesting question. There is no need to say that we do not know why dinosaurs disappeared. Is it necessary to formulate such facts of life in mathematical terms? Is it necessary that physicists play with models?

My answer is certainly, "yes", because we approach natural phenomena with a specific mind, and want to understand how nature (at large) works. And because we control our approximations.

During a seminar at the Faculty of Economy in Liège I was asked whether we had ever predicted a crash which had not occurred. The question is remarkable, and shows how poorly physics, and physics research is perceived by economists and other so-called 'soft scientists'. How could it occur that a model be used by a physicist in order to obtain a nonsense value? This is the first rule of the game we learn in a classroom: first, get the sign and the order of magnitude right. You next have to ask for the rough data and trust your experimentalist. It's the same thing in econophysics. Get the right data at seconds, minutes, hours, days, weeks intervals. Then do some statistical physics. All this shows that the right direction consists of the need for further investigations, about 'universality classes', and simple claims with too simple models (simple stops after 'spin glasses'). Also, expect field theory and ergodicity to come in. There will be too many models, but we will automatically organize them through social pressure; we will be led (I say with tongue in cheek) to the most interesting thus best funded questions. Money. Money. Money.

Finally, the robustness of models is a fundamental question. Models should be built, should be predictive (that is the fun part) and should be tested. Personally I am not sure that all models will stand up easily when they encompass more than one stock, more than one field, more than one dimension etc.

The need for all this can be questioned. The work cannot. Its intellectual and social values are obvious. A stable world is not the most interesting one, I agree, but it helps to sort out boundaries, and ranges in order to make progress feasible. Economies like life are still progressing, like sandpiles at the edge of chaos. That is quite a legal university research topic for physicists.
Reply...

My feature article is by no means a review of the emerging field of econophysics. Perhaps no one could write a review. Nothing is conclusive yet, so who would want to?

The feature is also not about our group's current work. And it is just a tiny part of a vast field. Through some very simple examples I tried to illustrate some model building ideas, hardly touching on economics or finance.

I agree with Ausloos' point of view that every physicist in econophysics has his/her personal approach to a personal choice of problems. This reinforces my view that the current state of affairs is that diversity of opinions prevails: you ask 10 physicists and you may get more than 10 opinions.

Precisely because of the lack of a generally agreed framework everybody is led by his own taste. Ausloos is certainly more optimistic than me, his list of success stories covers virtually all walks of economy and is impressive. The famous Belgian natural scientist, Nobel laureate Ilya Prigogine, would certainly approve such an optimistic, general approach. I share his enthusiasm for new challenges; they are exciting and physicists can contribute to many aspects. However, I personally feel that caution has to be exercised here since under the term 'econophysics' almost anything goes.

Our enthusiasm will be a lasting one if we do concrete work, analysing the mechanism piece by piece. Maybe one day it will suddenly dawn on us that a coherent picture has emerged. Fancy words like 'fractality' are already popular among the avant garde economists (who hold all kinds misconceptions), but looking into the substance can be disappointing. In short, physicists have long cherished the tradition of serious, concrete and objective work. Theory and experiment should go hand in hand. I believe that if we have some edge over traditional economists and philosophers on some aspects (I emphasize only in some aspects) it is because of our solid training and model building experience. Even econophysics will remain a 'soft science'; concrete work is still much in need. Yi-Cheng Zhang

Marcel Ausloos would like to acknowledge the enthusiastic comments and constructive support of $N$. Vandewalle, and the heartfelt interest of E. Labie, A. Minguet and Ph. Boveroux, notwithstanding the critical watch of J.P. Bouchaud, D. Stauffer and H.E. Stanley

\section{References}

[1] Y. C. Zhang, page 51; [2] B.B. Mandelbrot, The Fractal Geometry of Nature; W.H. Freeman, New York, 1982; [3] H.O. Peitgen and P.H. Richter, The Beauty of Fractals; Springer Verlag, Berlin, 1986; [4] P. Bak, How Nature Works; Copernicus, New York, 1996; [5] M. Ausloos and N. Vandewalle Europhys. News 26 (1995) 55; ibid. in Annual Reviews of Computational Physics D. Stauffer, Ed.; World Scientific, Singapore, 1996345 ; [6] O. Malcai, D. A. Lidar, O. Biham and D. Avnir, Phys. Rev. E 56 (1997) 2817; [7] G. K. Zipf, Human Behavior and the Principle of Least Effort; Addison Wesley, Cambridge, 1949; [8] N. Vandewalle and M. Ausloos, J. Phys. I (France) 5 (1995) 1011 ; ibid. Physica D 90 (1996) 262. [9] P. Bak, C. Tang and K. Wiesenfeld Phys. Rev. Lett. 59 (1987) 381 ; [10] D. Sornette Phys. Rep. (in press); [11] D. Stauffer and D. Sornette Physica A, in press; N. Vandewalle and M. Ausloos, in press; [12] H. G. Schuster, Deterministic Chaos; Physik Verlag, Weinheim, 1984; [13] D. Sornette, A. Johansen, and J. P. Bouchaud J. Phys. I (France) 6 (1996) 327; [14] M. Ausloos, Ph. Boveroux, A. Minguet, and N. Vandewalle, submitted for publication; [15] N. Vandewalle and M. Ausloos, submitted for publication; [16] P. M. Chaikin and T. C. Lubensky, Principles of Condensed Matter Physics; Cambridge Univ. Press, Cambridge, 1995; [17] song by Abba; [18] M. Ausloos, I. Mroz, A. Pekalski and N. Vandewalle Physica A 248 (1997) 155; [19] D. Stauffer, private communication; H. E. Stanley, private communication; [20] Int. J. Theor. Appl. Fin.; World Scientific, Singapore, 1997; [21] B. B.

Mandelbrot J. Business 36 (1963) 349; J. P. Bouchaud and D. Sornette, J. Phys. I (France) 4 (1994) 863; M. Levy, H. Levy and S. Solomon, Econ. Lett. 45 (1994) 103; J.A. Feigenbaum and P. G. O. Freund, Int. J. Mod. Phys. B10 (1996) 3737; [22] M. Ausloos and N. Vandewalle, submitted for publication; [23] E. E. Peters, Fractal Market Analysis (J. Wiley, New York, 1994); [24] R.M. Mantegna and H.E. Stanley, Nature 376 (1995) 46; [25] C.-K. Peng, S.V. Buldyrev, S. Havlin, M. Simmons, H.E. Stanley and A.L. Goldberger, Phys. Rev. E 49 (1994) 1685; [26] N. Vandewalle and M. Ausloos, Physica A 246 (1997) 454; [27] M. Ausloos and N. Vandewalle, submitted for publication; [28] J. P. Bouchaud, private communication; [29] H. Dupuis, in Trends and Tendencies 38 (1997) 26; ibid. 44 (1997) 11; [30] G. Legrand, in Cash 38 (1997) 3.

\section{Nicolas Vandewalle is launching a new course on Physics Models of Economies at the University of Liège, Belgium this year}

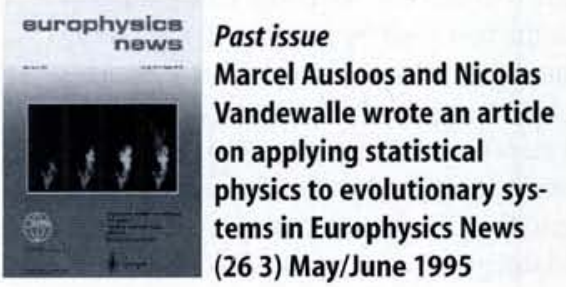

